\title{
Quality of Service in Cognitive Radio Network: Issues and challenges
}

\author{
NISAR A. LALA ${ }^{1 *}$, G. M. MIR ${ }^{1}$, ALTAF A. BALKHII and R. A. SIMNANI ${ }^{2}$ \\ ${ }^{1}$ College of Agricultural Engineering and Technology, Sher-e-Kashmir University of Agricultural \\ Sciences \& Technology of Kashmir Srinagar, J\&K, India. \\ ${ }^{2}$ Department of Electronics, Gandhi Memorial College Srinagar, J\&K, India.
}

\begin{abstract}
Cognitive radio $(C R)$ is a novel technology to resolve the issue of underutilization of wireless spectrum. There exists number of challenges and issues in designing and implementation of the cognitive radio. Extending qualityof-service (QoS) enabled applications to CR network is even more difficult task due to non-availability of the dedicated allocation of idle spectrum. CR imposes peculiar and unique challenges to guarantee quality of service of diverse flows in contrast to other wireless networks. This paper identifies the issues and challenges of QoS provisioning in cognitive radio networks.
\end{abstract}

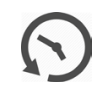

Article History

Received: 25 January 2018

Accepted:15 February 2018

\section{Keywords}

Cognitive radio, Quality of service (QoS), Spectrum management.

\section{Introduction}

The spectrum is a range of electromagnetic radiations that enables wireless communication and is managed by government. Day by day, the number of new wireless users and applications are increasing at a very rapid pace and particularly last decade has seen tremendous growth of both. Wireless users worldwide have reached 3.2 billion and were predicted to increase by 100 folds by $2013^{1}$. New applications demand additional allocation of spectrum which is not available and has created artificial scarcity of new allocation of spectrum. The first utilization survey reported that the allocated spectrum remained idle most of the time ${ }^{2}$ and is also function of geographical location and time. Other spectrum occupancy measurements conducted have revealed that the average utilization in New York City $^{3}$ was at $5.2 \%$ while in Chicago ${ }^{4}$ at $17.4 \%$. Several other countries spectrum occupancy measurement studies, such as Spain ${ }^{5}$, Singapore ${ }^{6}$, Germany ${ }^{7-8}$, New Zealand ${ }^{9}$ and United Kingdom ${ }^{10}$ also confirmed that spectrum is mostly under-utilized at this moment. The solution to decrease the under-utilization of the allocated spectrum is the cognitive radio $(\mathrm{CR})$. $\mathrm{CR}$ is defined as an intelligent device ${ }^{11}$ that is fully aware of its environment and takes decisions with an aim to realize maximum and efficient utilization of wireless spectrum. Based on the information extracted

CONTACT NISAR A LALA Ialanisar_ae@rediffmail.com 9 College of Agricultural Engineering and Technology, Sher-e-Kashmir University of Agricultural Sciences \& Technology of Kashmir Srinagar, J\&K, India

(C) 2018 The Author(s). Published by Oriental Scientific Publishing Company

This is an 6 Open Access article licensed under a Creative Commons Attribution-NonCommercial-ShareAlike 4.0 International License (https://creativecommons.org/licenses/by-nc-sa/4.0/), which permits unrestricted NonCommercial use, distribution, and reproduction in any medium, provided the original work is properly cited.

To link to this article: http://dx.doi.org/10.13005/ojcst11.01.07 
from the external world, CR alters its transmission parameters in order to adapt to the time varying environment ${ }^{12}$.

\section{Cognitive Radio}

The main purpose of the cognitive radio $(C R)$ is to make dynamic access to the idle spectrum a reality in order to have communication. A typical scenario consists of $\mathrm{CRs}$, those co-exist with the primary users (PUs). PU has the first priority to use the spectrum while CRs can use the spectrum opportunistically when the PU is not using the spectrum at that time. Main functions of CR are classified as under.

\section{Spectrum Sensing}

Spectrum sensing is the important function of the $\mathrm{CR}$. The CR has the capability to sense the radio environment in order to find idle channels known as spectrum hole and use those idle channels for its transmission. The sensing operation should also have the ability to detect the presence of the PU accurately when the later starts its communication again ${ }^{13}$.

\section{Spectrum Management}

Spectrum management helps in acquiring the best idle hole for the transmission of $\mathrm{CR}$ among large number of available holes found through the operation of sensing. Based on the availability of the spectrum, the suitable hole is allocated to the CR.

\section{Spectrum Mobility}

Spectrum mobility is referred as changing the frequency band during data transmission due to presence of PU on that band. The CR has to switch to another idle hole which is not used by the PU at that time in order to continue its transmission ${ }^{14}$. As soon as the PU needs the current frequency band, the CR has to terminate its transmission and free the presently occupied hole for the PU functioning ${ }^{15}$.

\section{Spectrum Sharing}

Spectrum sharing is an important functionality of CR as it coordinates the traffic between CRs and PUs. It is a challenging task as it requires high degree of cooperation, understanding and coordination between the two.

\section{Quality of Service}

Over the last decade, the development of wireless communication technology has shown exponential increase and wireless services have evolved from traditional voice service to a wide range of multimedia services. The bandwidth requirement varies from application to application. Hence, providing QoS to these applications according to their requirements of bandwidth is a critical and challenging task. QoS is the performance level of a service offered by the network to the user in order to achieve deterministic behavior by proper utilization of the network resources. The meaning of QoS changes as per the requirements of the application field. It is the process by which the performance and reliability of a network is controlled. It is a complex process and the modeling of QoS is a tough and difficult task. ISO 9000 defined QoS as "the degree to which a set of inherent characteristics fulfils requirements". ITU-T"16-17 defined QoS as "the collective effect of service performance which determines the degree of satisfaction of a user of the service". IETF ${ }^{18}$ considers QoS as "the ability to segment traffic or differentiate between traffic types in order to enable the network to treat certain traffic flows differently from others". The authors of ${ }^{19}$ identified three types of QoS such as Intrinsic QoS, Perceived QoS and Assessed QoS. Intrinsic QoS depends on the performance of the network and is described in terms of objective parameters such as delay, jitter, loss etc. Perceived QoS is the quality perceived by the users and is measured by their average opinion. Assessed QoS refers to the willingness of the users to continue the use of the specific service.

\section{Challenges of Qos Provisioning in CR}

The resources available to $\mathrm{CRs}$ in cognitive radio are highly dynamic and the QoS should be met without violating the priority right of PUs. QoS provisioning in cognitive radio is a challenging and highly difficult task. The requirement of QoS depends upon the nature of flows and the type of the networks. Cognitive radio imposes unique challenges for providing QoS and the identified issues for QoS provisioning are described as under. 


\section{Resource Identification}

The bandwidth of wireless networks is scarce than that of wire-line networks due to the physical limitation of wireless media. But the problem becomes even worse in cognitive radio as there is no fixed and dedicated allocation of spectrum for its functioning. $\mathrm{CR}$ has to find the idle hole to be used for its operation which is not being used by the PU at that time $20,21,22$. Therefore, the number of idle holes available at any instant is a function of time and varies with the activities of the primary users. Transmission of the CR is allowed only if idle holes are available. If no idle hole is found, blackouts may occur which introduce a peculiar challenge for QoS provisioning ${ }^{23,24}$.

\section{Wireless Channel Characteristics}

The wireless is a broadcast medium. The radio waves suffer from several impairments such as attenuation, thermal noise, interference, multipath fading and shadowing etc. ${ }^{25,26}$ during propagation through the wireless medium from transmitter to receiver ${ }^{27,28}$. These factors increase the variability of the some of the parameters responsible for providing the QoS. In cognitive radio, due to switching of operating channel, these impairments also change which results in change of channel characteristics. As a result, this puts additional challenges for QoS provisioning.

\section{Sensing Duration}

Detection of the primary users is performed through spectrum sensing and sensing duration is the critical parameter for QoS provisioning in cognitive radio $29,30,31$. During sensing phase, data transmission is stopped. Sensing time is the function of interference in the channel and may increase with increase in interference ${ }^{32}$. As a result, this creates the varying jitter between the data packets. The sensing time should be of such duration that it is able to scan the whole spectrum and at the same time it doesn't affect the QoS of the application running over the $\mathrm{CR}$. Therefore, maintaining a trade-off between the two is a challenging task $\mathrm{k}^{24,33,34}$.

\section{Handoff Management}

Due to the arrival of the primary user on the channel presently occupied by the $\mathrm{CR}$, there is a need to change the operating channel. Therefore, the spectrum mobility imposes additional delay due to instantaneous sensing of the spectrum in order to find idle channels to continue the ongoing communication. The movement from one frequency channel to another imposes further challenges of change in channel characteristics and may also introduce variations in the bandwidth and noise levels. The previously existing channel conditions will not be valid in the new operating frequency channel. As a result, there will be change in certain parameters (such as delay, jitter, SNIR, loss etc.) and those parameters have strong influence on QoS provisioning ${ }^{23,33,35}$.

\section{Handoff Rate}

Experimental studies have shown that cost of channel switching is significant in wireless mesh networks ${ }^{36}$ and channel switching causes non-negligible interruption in the connectivity among nodes which can be of the order of 10 seconds $^{37}$. In cognitive radio, frequent channel switching due to spectrum mobility takes place which will make the situation even worse and will result in QoS degradation of the CRs. Hence, reduction in switching (or handoff) rate is a critical and challenging task.

\section{Interference Management}

Sensing phase is followed by data transmission phase in cognitive radio. Interference ${ }^{38}$ in the communication channel is introduced due to missed detection which occurs due to low signal-to-noiseratio (SNR) or hidden terminal problem of the primary user. As a result, collision of data packets takes place between CR and the PU which increases the loss rate of the transmission and at the same time increases the interference to the primary user. As a result, there will be decrease in the QoS. Therefore, maintenance of the assured level of QoS becomes a challenging task ${ }^{11,23-24,39-40}$.

\section{Conclusion}

The resource availability is highly dynamic in CR and thus, providing the QoS as per the requirements of the flow is a challenging issue. This paper introduced the challenges of QoS provisioning in the CR and the identified issues for the QoS provisioning. The identified issues are; resource identification, wireless channel characteristics, sensing duration, handoff management, handoff rate and interference 
management. These issued needs to be resolved before quality of service provisioning becomes reality in CR networks.

\section{Acknowledgement}

I would like to acknowledge and express my heartiest gratitude to my Ph. D supervisor Professor MoinUddin who introduced me to the very interesting subject "Cognitive Radio Technology". I have benefitted tremendously from his vision, technical insight and valuable suggestions. Prof. Moin Uddin is a senior member, IEEE. He has more than 35 years of experience in academics and research and has served at key Positions as Director, NIT Jallandhar, Pro-Vice-Chancellor, Delhi Technological University.

\section{References}

1. Cisco. Scaling the mobile internet. White Paper, 2009.

2. Federal Communications Commission (FCC). Notice of proposed rulemaking and order No. 03-222. Dec. 2003.

3. Spectrum occupancy measurements (SSC). 1595 Spring Hill Rd, Suite 110, Vienna, VA 22182, USA, Tech Rep.. 2005.

4. McHenry M.A., Tenhule P.A., McCloskey D., Roberson D.A., Hood C.S. Chicago spectrum occupancy measurements and analysis and a long term studies proposal. Proceedings of $1^{\text {st }}$ International Workshop on Technology and Policy for Accessing Spectrum (TAPAS' 06) New York, USA (ACM), 2006.

5. Lopez-Benitez M., Umbert A., Casadevall F. Evaluation of spectrum occupancy in Spain for cognitive radio applications. Proceedings of IEEE $69^{\text {th }}$ Vehicular Technology Conference (VTC 2009 Spring), 2009; 1-5.

6. Islam M.H., Koh C.L., Oh S.W., Qing X., Lai Y.Y., et al., Spectrum survey in Singapore: Occupancy measurements and analyses. Proceedings of $3^{\text {rd }}$ International Conference on Cognitive Radio Oriented Wireless Networks and Communications (CROWNCOM), May 2008;1-7.

7. Wellens M., Wu J., Mahonen P. Evaluation of spectrum occupancy in indoor and outdoor scenario in the context of cognitive radio. Proceedings of $2^{\text {nd }}$ International Conference on Cognitive Radio Oriented Wireless Networks and Communications (CROWNCOM), Aug 2007; 420-7.

8. Harrold T.J., Cepeda R.A., Beach M.A. Long-term measurements of spectrum occupancy characteristics. Proceedings of
IEEE International Symposium on Dynamic Spectrum Access Networks (DySpan) Aachen, Germany, May 2011; 83-9.

9. Chiang R.I.C., Rowe G.B., Sowerby K.W. A quantitative analysis of spectral occupancy measurements for cognitive radio. Proceedings of IEEE $65^{\text {th }}$ Vehicular Technology Conference (VTC), Dublin, Ireland, April 2007; 3016-20.

10. Mehdawi M., Riley N., Paulson K., Fanan A., Ammar M. Spectrum occupancy survey in Hull-UK for cognitive radio applications: Measurement and analysis. J. Scientific and Technology Research, April 2013; 2(4): 231-6.

11. Haykin S. Cognitive radio: Brain empowered wireless communications. IEEE Journal on Selected Areas in Communications, 2005; 23(2):201-20.

12. Federal Communication Commissi on (FCC). Unlicensed operation in TV broadcast bands. Notice for Proposed Rule Making, ET Docket No. 04-113, May 2004.

13. Arslan H. Cognitive radio, software defined radio, and adaptive wireless systems. Springer, 2007(e-book).

14. Fu X., Zhou W., Xu J., Song J. Extended mobility management challenges over cellular networks combined with cognitive radio by using multi-hop network. Proceedings of International Conference on Software Engineering, Artificial Intelligence, Networking, and Parallel/distributed Computing, July 2007; 2:683-8.

15. Liu H. J., Wang Z. X., Li S. F., Yi M. Study on the performance of spectrum mobility in cognitive wireless network. Proceedings 
of $11^{\text {th }}$ IEEE International Conference on Communication Systems (ICCS), 2008: 1010-4.

16. ITU-T Recommendations. Terms and definitions related to Quality of Service and network performance including dependability. ITU-T Recommendation E.800, August 1994.

17. ETSI. Network aspects (NA): general aspects of Quality of Service and network performance. ETSI Technical Report, ETR 003, $2^{\text {nd }}$ Edition, October 1994.

18. ETSI. Satellite earth stations and systems, broadband satellite multimedia IP. IP Internet working over Satellite: Performance, Availability and Quality of Service, March 2003, ETSI Technical Report.

19. Hardy W. C. QoS measurement and evaluation of telecommunications Quality of Service. John Wiley and Sons, England, 2001.

20. Nguyen D., Tran L., Pirinen P., and Latvaaho M. On the spectral efficiency of full-duplex small cell wireless systems. IEEE Trans. Wireless Communication, Sept. 2014; 13(9): 4896-910.

21. Lu X., Wang P., Niyato D., Kim D.I., and Han Z. Wireless Networks with RF Energy Harvesting: A Contemporary Survey. IEEE Communications Surveys \& Tutorials, May 2015; 17(2):757-89.

22. Hoang D.T., Niyato D., Wang P., and Kim D. I. Opportunistic Channel Access and RF Energy Harvesting in Cognitive Radio Networks. IEEE Trans. on Selected Areas in Communications, Nov. 2014; 32(11): 1-14.

23. Akyildiz I.F., Lee W.Y., Vuran M.C., Mohanty S. Next generation/dynamic spectrum access/cognitive radio wireless networks: A survey. Computer Networks (Elsevier), 2006; 50:2127-59.

24. Attar A., Ghorashi S. A., Sooriyabandara M. and Aghvami A.H. Challenges of real-time secondary usage of spectrum. Computer Networks (Elsevier), 2008; 52 (4): 816-30.

25. Boulogeorgos A.-A. A., Sofotasios P. C., Selim B., Muhaidat S., Karagiannidis G. K., and Valkama M. Effects of RF impairments in communications over cascaded fading channels. IEEE Transactions on Vehicular Technology, 2016; 65(11): 1-17.
26. Li B., Sun M., Li X., Nallanathan A., and Zhao C. Energy Detection based Spectrum Sensing for Cognitive Radios over Time-Frequency Doubly Selective Fading Channels. IEEE Transactions on signal processing, Jan. 2015; 63(2): 402-17.

27. Walke B. H. Mobile Radio Networks: Networking and protocols. $2^{\text {nd }}$ Edition, 1999.

28. Garg K. Wireless Networks Evolution: 2G to 3G. Reprint 2003.

29. Liao Y., Wang T., Song L., Han Z. Listenand-Talk: Protocol Design and Analysis for Full-duplex Cognitive Radio Networks. IEEE Trans. on Vehicular Technology, Jan. 2017; 66(1): 656-67.

30. Sharma S. K., Bogale T. E., Le L. B., Chatzinotas S., Wang X., Ottersten B. TwoPhase Concurrent Sensing and Transmission Scheme for Full Duplex Cognitive Radio. in Proc. IEEE VTC Spring, Sept. 2016.

31. Altrad O., Muhaidat S., Al-Dweik A. S., and Yoo P. Opportunistic spectrum access in cognitive radio networks under imperfect spectrum sensing. IEEE on Vehicular Technology, Feb. 2014; 63(2): 920-5.

32. Lee W. Y. and Akyildiz I. F. Optimal spectrum sensing framework for cognitive radio networks. IEEE Transactions on Wireless Communications, 2008; 7(10): 3845-57.

33. Qiao X., Tan Z. and Li J. Combined optimization of spectrum handoff and spectrum sensing for cognitive radio systems. $7^{\text {th }}$ IEEE International Conference on Wireless Communications, Networking and Mobile Computing (WiCOM), 2011:1-4.

34. Ghasemi A. and Sousa E. S. Spectrum sensing in cognitive radio networks: requirements, challenges and design trade-offs. IEEE Communications Magazine, April 2008; 46(4): 32-9.

35. Baroudi U. and Alfadhly A. Effects of mobility and primary appearance probability on spectrum handoff. $73^{\text {rd }}$ IEEE Vehicular Technology Conference (VTC spring), 2011: 1-6.

36. Li P., Scalabrino N. and Fang Y. How to effectively use multiple channels in wireless mesh networks. IEEE Transactions on Parallel and Distributed Systems, 2009; 20(11): 1641 52. 
37. Avallone S. and Stasi G. D. An experimental study of the channel switching cost in multichannel wireless mesh networks. IEEE Communications magazine, Sep. 2013; 51(9): 124-34.

38. Afifi W. and Krunz M. Incorporating selfinterference suppression for full-duplex operation in opportunistic spectrum access systems. IEEE Trans. Wireless Commun., Apr. 2015;14(4): 2180-91.
39. Rabbachin A., Quek T. Q. S., Shin H. and Win M. Z. Cognitive network interference. IEEE Journal on Selected Areas in Communications, 2011; 29(2): 480-93.

40. Xing Y. and Chandramouli R. QoS constrained secondary spectrum sharing. IEEE International Symposium on Dynamic Spectrum Access Networks (DySPAN), Nov. 2005: 658-61. 